1 Hacettepe Journal of Mathematics and Statistics

$\bigcap$ Volume 47 (6) (2018), 1521-1536

\title{
Existence results for nonlinear boundary value problems with $m$-point integral boundary condition
}

\author{
Tugba Senlik Cerdik ${ }^{* \dagger}$ and Nuket Aykut Hamal ${ }^{\ddagger}$
}

\begin{abstract}
In this paper, we investigate the existence of positive solutions for the nonlinear $m$-point boundary value problems with integral boundary condition. By using fixed-point index theorem and Leggett- Williams fixed point theorem, the existence and multiplicity of positive solutions are obtained. As an application, two examples are given to demonstrate our results.
\end{abstract}

Keywords: Boundary value problems, fixed point theorems, integral boundary conditions.

Mathematics Subject Classification (2010): 34B10, 34B15

Received: 27.06.2016 Accepted : 06.02.2017 Doi : 10.15672/HJMS.2017.450

\section{Introduction}

Nonlinear boundary value problems for ordinary differential equations (ODEs) with nonlocal boundary conditions (BCs) have been well studied over the past decades. The study of nonlocal BCs for ODEs goes back, as far as we know, to Picone [12] and has been widely developed during the years. We refer the reader to the reviews by Whyburn [16], Conti [4] and Ntouyas [11]. Some important contributions were given by Bitsadze and Samarskii [2].

At the same time, boundary value problems (BVPs) with Riemann-Stieltjes integral boundary conditions include two point, three-point, multi-point and the Riemann integral BVPs as special cases. Such BVPs have attracted the attention of researchers such as $[14,15,1,5,6,7,9,10,13,17,18,3]$.

In [6], Feng et al. considered the second order differential equation

\footnotetext{
*Department of Mathematics, Ege University, Izmir, Turkey, Email: tubasenlik@gmail.com $\dagger_{\text {Corresponding Author. }}$

$\ddagger$ Department of Mathematics, Ege University, Izmir, Turkey, Email: nuket .aykut@ege.edu.tr
} 


$$
\left\{\begin{array}{l}
u^{\prime \prime}(t)+f(t, u(t))=\theta, \quad t \in(0,1), \\
u(0)=\int_{0}^{1} g(t) u(t) d t, \quad u(1)=\theta \\
\text { or } u(0)=\theta, \quad u(1)=\int_{0}^{1} g(t) u(t) d t .
\end{array}\right.
$$

They investigated the existence and nonexistence of positive solutions for a class of nonlinear boundary value problem of the second-order differential equations with integral boundary conditions in ordered Banach spaces. The arguments were based upon a specially constructed cone and the fixed point theory in a cone for strict set contraction operators.

In [13], Tariboon and Sudsutad considered second-order $m$-point integral boundary value problem

$$
\left\{\begin{array}{l}
u^{\prime \prime}(t)+a(t) f(t)=0, \quad t \in(0,1), \\
u(0)=0, \quad u(1)=\sum_{i=1}^{m-1} \alpha_{i} \int_{\eta_{i-1}}^{\eta_{i}} u(s) d s .
\end{array}\right.
$$

By using the Guo-Krasnoselskii's fixed point theorem, they obtained the existence criteria of at least one positive solution if $\mathrm{f}$ is either superlinear or sublinear.

In $[14,15]$, Webb and Infante used fixed point index theory and gave a general method for solving problems with integral BCs of Riemann-Stieltjes type. In [15], they studied the existence of multiple positive solutions of nonlinear differential equations of the form

$$
\left\{\begin{array}{l}
-u^{\prime \prime}(t)=g(t) f(t, u(t)), \quad t \in(0,1), \\
u(0)=\alpha[u], \quad u(1)=\beta[u], \\
\text { or } u(0)=\alpha[u], \quad u^{\prime}(1)=\beta[u], \\
\text { or } u(0)=\alpha[u], \quad u^{\prime}(1)+\beta[u]=0, \\
\text { or } u^{\prime}(0)=\alpha[u], \quad u(1)=\beta[u], \\
\text { or } u^{\prime}(0)+\alpha[u]=0, \quad u(1)=\beta[u] .
\end{array}\right.
$$

Here $\alpha[u], \beta[u]$ are bounded linear functionals on $\mathcal{C}[0,1]$ given by

$$
\alpha[u]=\int_{0}^{1} g(t) u(s) d A(s), \quad \beta[u]=\int_{0}^{1} g(t) u(s) d B(s)
$$

with $A, B$ functions of bounded variation.

Motivated by the results above, in this paper, we consider the following second order $m$-point integral boundary value problem (BVP)

$$
\left\{\begin{array}{l}
u^{\prime \prime}(t)+f(t, u(t))=0, \quad t \in(0,1) \\
\beta u(0)-\gamma u^{\prime}(0)=0, \quad u(1)=\sum_{i=1}^{m-1} \alpha_{i} \int_{\eta_{i-1}}^{\eta_{i}} u(s) d s .
\end{array}\right.
$$

Here the boundary conditions of (1.1) are a special case of Riemann-Stieltjes integral. We get the existence and multiplicity of positive solutions for the BVP (1.1) by using the fixed point index theorem and the Leggett-Williams fixed point theorem.

We will assume that the following assumptions are satisfied:

(C1) $f \in \mathcal{C}\left([0,1] \times \mathcal{R}^{+}, \mathcal{R}^{+}\right), \mathcal{R}^{+}=[0,+\infty), 0=\eta_{0}<\eta_{1}<\ldots<\eta_{m-2}<\eta_{m-1}=1$;

(C2) $\beta, \gamma \geq 0, \beta+\gamma>0, \alpha_{i} \geq 0$ for $i=1,2, \ldots, m-1, \sum_{i=1}^{m-1} \alpha_{i} \in(0,1)$ and $d>0$ 
where

$$
d=\beta\left(2-\sum_{i=1}^{m-1} \alpha_{i}\left(\eta_{i}^{2}-\eta_{i-1}^{2}\right)\right)+2 \gamma\left(1-\sum_{i=1}^{m-1} \alpha_{i}\left(\eta_{i}-\eta_{i-1}\right)\right)
$$

We note that the $m$-point boundary condition is related to $m-1$ intervals of the area under the curve of solution $u(t)$ from $t=\eta_{i-1}$ to $t=\eta_{i}$ for $i=1, \ldots, m-1$.

The organisation of the paper is as follows. In Section 2, we present some necessary lemmas that will be used to prove our main results. In Section 3, we apply fixed point theorems to obtain the existence of solutions for the BVP (1.1) and we give examples to illustrate our results.

\section{Preliminaries}

In this section, we will employ several lemmas to prove the main results in this paper.

2.1. Lemma. Let $\alpha_{i} \geq 0$ for $i=1,2, \ldots, m-1$, and $d \neq 0$. If $y \in \mathcal{C}[0,1]$, then the problem

$$
\begin{gathered}
u^{\prime \prime}(t)+y(t)=0, \quad t \in(0,1), \\
\beta u(0)-\gamma u^{\prime}(a)=0, \quad u(1)=\sum_{i=1}^{m-1} \alpha_{i} \int_{\eta_{i-1}}^{\eta_{i}} u(s) d s,
\end{gathered}
$$

has a unique solution

$$
\begin{aligned}
u(t)= & -\int_{0}^{t}(t-s) y(s) d s+\frac{2(\gamma+\beta t)}{d} \int_{0}^{1}(1-s) y(s) d s \\
& -\frac{\gamma+\beta t}{d} \sum_{i=1}^{m-1} \alpha_{i} \int_{0}^{\eta_{i}}\left(\eta_{i}-s\right)^{2} y(s) d s \\
& +\frac{\gamma+\beta t}{d} \sum_{i=1}^{m-1} \alpha_{i} \int_{0}^{\eta_{i-1}}\left(\eta_{i-1}-s\right)^{2} y(s) d s .
\end{aligned}
$$

Proof. We have from (2.1),

$$
u^{\prime \prime}(t)=-y(t) \text {. }
$$

It is easy to see by integration of both sides of $(2.1)$ on $[0, t]$,

$$
u^{\prime}(t)=u^{\prime}(0)-\int_{0}^{t} y(s) d s .
$$

Integrating again, we can get

$$
u(t)=u(0)+u^{\prime}(0) t-\int_{0}^{t}(t-s) y(s) d s .
$$

From condition (2.2)

$$
u(t)=-\int_{0}^{t}(t-s) y(s) d s+u^{\prime}(0)\left(\frac{\gamma}{\beta}+t\right) .
$$

So

$$
u(1)=-\int_{0}^{1}(1-s) y(s) d s+u^{\prime}(0)\left(\frac{\gamma}{\beta}+1\right)
$$


Integrating (2.3) from $\eta_{i-1}$ to $\eta_{i}$ for $0 \leq \eta_{i-1} \leq \eta_{i} \leq 1, i=1, \ldots, m-1$ and reversing the order of double integrating, we get

$$
\begin{aligned}
\int_{\eta_{i-1}}^{\eta_{i}} u(s) d s= & -\int_{\eta_{i-1}}^{\eta_{i}}\left(\int_{0}^{r}(r-s) y(s) d s\right) d r \\
& +u^{\prime}(0) \frac{\gamma}{\beta}\left(\eta_{i}-\eta_{i-1}\right)+u^{\prime}(0) \frac{\left(\eta_{i}^{2}-\eta_{i-1}^{2}\right)}{2} \\
= & -\int_{0}^{\eta_{i}} \frac{\left(\eta_{i}-s\right)^{2}}{2} y(s) d s+\int_{0}^{\eta_{i-1}} \frac{\left(\eta_{i-1}-s\right)^{2}}{2} y(s) d s \\
& +u^{\prime}(0) \frac{\gamma}{\beta}\left(\eta_{i}-\eta_{i-1}\right)+u^{\prime}(0) \frac{\left(\eta_{i}^{2}-\eta_{i-1}^{2}\right)}{2} .
\end{aligned}
$$

From condition (2.2), we have

$$
\begin{aligned}
-\int_{0}^{1}(1-s) y(s) d s+u^{\prime}(0)\left(\frac{\gamma}{\beta}+1\right)= & -\frac{1}{2} \sum_{i=1}^{m-1} \alpha_{i} \int_{0}^{\eta_{i}}\left(\eta_{i}-s\right)^{2} y(s) d s \\
& +\frac{1}{2} \sum_{i=1}^{m-1} \alpha_{i} \int_{0}^{\eta_{i-1}}\left(\eta_{i-1}-s\right)^{2} y(s) d s \\
& +u^{\prime}(0) \sum_{i=1}^{m-1} \alpha_{i}\left(\frac{\gamma}{\beta}\left(\eta_{i}-\eta_{i-1}\right)+\frac{\eta_{i}^{2}-\eta_{i-1}^{2}}{2}\right) .
\end{aligned}
$$

We obtain,

$$
\begin{aligned}
u^{\prime}(0)= & \frac{2 \beta}{d} \int_{0}^{1}(1-s) y(s) d s-\frac{\beta}{d} \sum_{i=1}^{m-1} \alpha_{i} \int_{0}^{\eta_{i}}\left(\eta_{i}-s\right)^{2} y(s) d s \\
& +\frac{\beta}{d} \sum_{i=1}^{m-1} \alpha_{i} \int_{0}^{\eta_{i-1}}\left(\eta_{i-1}-s\right)^{2} y(s) d s
\end{aligned}
$$

Hence, (2.1)-(2.2) has a unique solution

$$
\begin{aligned}
u(t)= & -\int_{0}^{t}(t-s) y(s) d s+\frac{2(\gamma+\beta t)}{d} \int_{0}^{1}(1-s) y(s) d s \\
& -\frac{\gamma+\beta t}{d} \sum_{i=1}^{m-1} \alpha_{i} \int_{0}^{\eta_{i}}\left(\eta_{i}-s\right)^{2} y(s) d s \\
& +\frac{\gamma+\beta t}{d} \sum_{i=1}^{m-1} \alpha_{i} \int_{0}^{\eta_{i-1}}\left(\eta_{i-1}-s\right)^{2} y(s) d s
\end{aligned}
$$

2.2. Lemma. Let $\sum_{i=1}^{m-1} \alpha_{i}<1$ and $d>0$. If $y \in \mathcal{C}([0,1],[0,+\infty))$, then unique solution $u$ of (2.1)-(2.2) satisfies $u(t) \geq 0$ for $t \in[0,1]$.

Proof. From the fact that $u^{\prime \prime}(t)=-y(t) \leq 0$, we know that the graph of $u$ is concave down on $[0,1]$. It suffices to prove that $u(0) \geq 0$ and $u(1) \geq 0$. 


$$
\begin{aligned}
u(0)= & \frac{2 \gamma}{d} \int_{0}^{1}(1-s) y(s) d s-\frac{\gamma}{d} \sum_{i=1}^{m-1} \alpha_{i} \int_{0}^{\eta_{i}}\left(\eta_{i}-s\right)^{2} y(s) d s \\
& +\frac{\gamma}{d} \sum_{i=1}^{m-1} \alpha_{i} \int_{0}^{\eta_{i-1}}\left(\eta_{i-1}-s\right)^{2} y(s) d s \\
\geq & \frac{2 \gamma}{d} \int_{0}^{1}(1-s) y(s) d s-\frac{2 \gamma}{d} \sum_{i=1}^{m-1} \alpha_{i} \int_{0}^{1}(1-s)^{2} y(s) d s \\
\geq & \frac{2 \gamma}{d} \int_{0}^{1}(1-s) y(s) d s-\frac{2 \gamma}{d} \sum_{i=1}^{m-1} \alpha_{i} \int_{0}^{1}(1-s) y(s) d s \\
= & \frac{2 \gamma}{d}\left(1-\sum_{i=1}^{m-1} \alpha_{i}\right) \int_{0}^{1}(1-s) y(s) d s \\
\geq & 0 .
\end{aligned}
$$

$$
\begin{aligned}
u(1)= & -\int_{0}^{1}(1-s) y(s) d s+\frac{2 \gamma+2 \beta}{d} \int_{0}^{1}(1-s) y(s) d s \\
& -\frac{\gamma+\beta}{d} \sum_{i=1}^{m-1} \alpha_{i} \int_{0}^{\eta_{i}}\left(\eta_{i}-s\right)^{2} y(s) d s \\
& +\frac{\gamma+\beta}{d} \sum_{i=1}^{m-1} \alpha_{i} \int_{0}^{\eta_{i-1}}\left(\eta_{i-1}-s\right)^{2} y(s) d s \\
= & \frac{\beta \sum_{i=1}^{m-1} \alpha_{i}\left(\eta_{i}^{2}-\eta_{i-1}^{2}\right)+2 \gamma \sum_{i=1}^{m-1} \alpha_{i}\left(\eta_{i}-\eta_{i-1}\right)}{d} \int_{0}^{1}(1-s) y(s) d s \\
& -\frac{\gamma+\beta}{d} \sum_{i=1}^{m-1} \alpha_{i} \int_{0}^{\eta_{i}}\left(\eta_{i}-s\right)^{2} y(s) d s \\
& +\frac{\gamma+\beta}{d} \sum_{i=1}^{m-1} \alpha_{i} \int_{0}^{\eta_{i-1}}\left(\eta_{i-1}-s\right)^{2} y(s) d s \\
\geq & \frac{\gamma+\beta}{d} \sum_{i=1}^{m-1} \alpha_{i} \int_{0}^{\eta_{i-1}}\left[\left(\eta_{i}^{2}-\eta_{i-1}^{2}\right)(1-s)-\left(\eta_{i}-s\right)^{2}+\left(\eta_{i-1}-s\right)^{2}\right] y(s) d s \\
& +\frac{\gamma+\beta}{d} \sum_{i=1}^{m-1} \alpha_{i} \int_{\eta_{i-1}}^{\eta_{i}}\left[\left(\eta_{i}^{2}-\eta_{i-1}^{2}\right)(1-s)-\left(\eta_{i}-s\right)^{2}\right] y(s) d s \\
& +\frac{\gamma+\beta}{d} \sum_{i=1}^{m-1} \alpha_{i} \int_{\eta_{i}}^{1}\left(\eta_{i}^{2}-\eta_{i-1}^{2}\right)(1-s) y(s) d s \\
\geq & \frac{\gamma+\beta}{d} \sum_{i=1}^{m-1} \alpha_{i} \int_{\eta_{i-1}}^{\eta_{i}}\left(\eta_{i}-\eta_{i-1}\right)\left(\eta_{i-1}(1-s)+s\left(1-\eta_{i}\right)\right) y(s) d s \\
& +\frac{\gamma+\beta}{d} \sum_{i=1}^{m-1} \alpha_{i} \int_{\eta_{i}}^{1}\left(\eta_{i}^{2}-\eta_{i-1}^{2}\right)(1-s) y(s) d s \\
&
\end{aligned}
$$


1526

$$
\begin{aligned}
& \geq \frac{\gamma+\beta}{d} \sum_{i=1}^{m-1} \alpha_{i} \int_{\eta_{m-2}}^{1}\left(\eta_{i}^{2}-\eta_{i-1}^{2}\right)(1-s) y(s) d s \\
& \geq 0 .
\end{aligned}
$$

The proof is complete.

2.3. Lemma. Assume that $0<\sum_{i=1}^{m-1} \alpha_{i}<1$ and $d>0$. If $y \in \mathcal{C}([0,1],[0,+\infty))$, then unique solution $u$ of (2.1)-(2.2) satisfies

$$
\inf _{t \in\left[\eta_{1}, 1\right]} u(t) \geq \Gamma\|u\|
$$

where,

$$
\begin{aligned}
& \Gamma=\min _{2 \leq s \leq m-2}\left\{\eta_{1}, \frac{1}{2} \sum_{i=1}^{m-2} \alpha_{i}\left(\eta_{i}^{2}-\eta_{i-1}^{2}\right),\right. \\
& \frac{\sum_{i=1}^{s-1} \alpha_{i}\left(\eta_{i}^{2}-\eta_{i-1}^{2}\right)+\sum_{i=s}^{m-2} \frac{\alpha_{i}}{\eta_{i}}\left(\eta_{i}^{2}-\eta_{i-1}^{2}\right)\left(1-\eta_{i}\right)}{2-\sum_{i=s}^{m-2} \alpha_{i}\left(\eta_{i}^{2}-\eta_{i-1}^{2}\right)}, \\
& \left.\frac{\sum_{i=1}^{m-2} \frac{\alpha_{i}}{\eta_{i}}\left(\eta_{i}^{2}-\eta_{i-1}^{2}\right)\left(1-\eta_{i}\right)}{2-\sum_{i=1}^{m-2} \alpha_{i}\left(\eta_{i}^{2}-\eta_{i-1}^{2}\right)}\right\}, \\
& \text { and }\|u\|=\max _{t \in[0,1]}|u(t)| \text {. }
\end{aligned}
$$

Proof. If $u(t)$ is maximum at $t=\bar{t}$, then $\|u\|=u(\bar{t})$. The proof is divided into four steps.

Step 1. If $\inf _{t \in\left[\eta_{1}, 1\right]} u(t)=u\left(\eta_{1}\right)$, then the concavity of $u$ implies that $u\left(\eta_{1}\right) \geq \eta_{1} u(\bar{t})$. Thus, $\inf _{t \in\left[\eta_{1}, 1\right]} u(t) \geq \Gamma\|u\|$.

Step 2. If $\bar{t} \leq \eta_{1}$ and $\inf _{t \in\left[\eta_{1}, 1\right]} u(t)=u(1)$, then the concavity of $u$ implies

$$
\frac{u\left(\eta_{i}\right)-u(1)}{1-\eta_{i}} \geq \frac{u(\bar{t})-u(1)}{1-\bar{t}} \geq u(\bar{t})-u(1), 1 \leq i \leq m-2 .
$$

So,

$$
u\left(\eta_{i}\right)-\eta_{i} u(1) \geq\left(1-\eta_{i}\right) u(\bar{t}), 1 \leq i \leq m-2 .
$$

Therefore,

$$
\begin{aligned}
& \frac{1}{2} \sum_{i=1}^{m-2} \frac{\alpha_{i}}{\eta_{i}}\left(\eta_{i}^{2}-\eta_{i-1}^{2}\right)\left(u\left(\eta_{i}\right)-\eta_{i} u(1)\right) \\
& \geq \frac{1}{2} \sum_{i=1}^{m-2} \frac{\alpha_{i}}{\eta_{i}}\left(\eta_{i}^{2}-\eta_{i-1}^{2}\right)\left(1-\eta_{i}\right) u(\bar{t}), \quad 1 \leq i \leq m-2 .
\end{aligned}
$$

We know that $u(t)$ from $t=\eta_{i-1}$ to $t=\eta_{i}$ for $i=1,2, \ldots, m-1$ satisfies 


$$
\int_{\eta_{i-1}}^{\eta_{i}} u(s) d s \geq \frac{1}{2}\left(\eta_{i}-\eta_{i-1}\right)\left(u\left(\eta_{i}\right)+u\left(\eta_{i-1}\right)\right) .
$$

where $\frac{1}{2}\left(\eta_{i}-\eta_{i-1}\right)\left(u\left(\eta_{i}\right)+u\left(\eta_{i-1}\right)\right)$ is the area of the trapezoid under the curve $u(t)$. From the concavity of $u$ and Lemma 2.2, we get

$$
\frac{u\left(\eta_{1}\right)}{\eta_{1}} \geq \frac{u\left(\eta_{2}\right)}{\eta_{2}} \geq \ldots \geq \frac{u\left(\eta_{i-1}\right)}{\eta_{i-1}} \geq \frac{u\left(\eta_{i}\right)}{\eta_{i}} \geq \ldots \geq \frac{u(1)}{1} .
$$

Then, using conditions (2.6) and (2.7), we have

$$
\begin{aligned}
u(1) & =\sum_{i=1}^{m-1} \alpha_{i} \int_{\eta_{i-1}}^{\eta_{i}} u(s) d s \\
& \geq \frac{1}{2} \sum_{i=1}^{m-1} \alpha_{i}\left(\eta_{i}-\eta_{i-1}\right)\left(u\left(\eta_{i}\right)+u\left(\eta_{i-1}\right)\right) \\
& \geq \frac{1}{2} \sum_{i=1}^{m-2} \alpha_{i}\left(\eta_{i}-\eta_{i-1}\right)\left(u\left(\eta_{i}\right)+u\left(\eta_{i-1}\right)\right) \\
& \geq \frac{1}{2} \sum_{i=1}^{m-2} \alpha_{i}\left(\eta_{i}-\eta_{i-1}\right)\left(u\left(\eta_{i}\right)+\frac{\eta_{i-1}}{\eta_{i}} u\left(\eta_{i}\right)\right) \\
& =\frac{1}{2} \sum_{i=1}^{m-2} \frac{\alpha_{i}}{\eta_{i}}\left(\eta_{i}^{2}-\eta_{i-1}^{2}\right) u\left(\eta_{i}\right) .
\end{aligned}
$$

i.e.,

$$
u(1) \geq \frac{1}{2} \sum_{i=1}^{m-2} \frac{\alpha_{i}}{\eta_{i}}\left(\eta_{i}^{2}-\eta_{i-1}^{2}\right) u\left(\eta_{i}\right) .
$$

Combining conditions (2.5), (2.8), we get

$$
u(1) \geq \frac{\sum_{i=1}^{m-2} \frac{\alpha_{i}}{\eta_{i}}\left(\eta_{i}^{2}-\eta_{i-1}^{2}\right)\left(1-\eta_{i}\right)}{2-\sum_{i=1}^{m-2} \alpha_{i}\left(\eta_{i}^{2}-\eta_{i-1}^{2}\right)} u(\bar{t}) .
$$

Thus, $\inf _{t \in\left[\eta_{1}, 1\right]} u(t) \geq \Gamma\|u\|$.

Step 3. If there exists $2 \leq s \leq m-2$ such that $\eta_{s-1}<\bar{t} \leq \eta_{s}$, and $\inf _{t \in\left[\eta_{1}, 1\right]} u(t)=u(1)$, for $s \leq i \leq m-2$,

$$
\frac{u\left(\eta_{i}\right)-u(1)}{1-\eta_{i}} \geq \frac{u(\bar{t})-u(1)}{1-\bar{t}}
$$

So,

$$
u\left(\eta_{i}\right)-\eta_{i} u(1) \geq\left(1-\eta_{i}\right) u(\bar{t}) \text { for } s \leq i \leq m-2 .
$$

In addition, in view of (2.7), we get

$$
u\left(\eta_{i}\right) \geq \eta_{i} u(\bar{t}) \text { for } 1 \leq i \leq s-1 .
$$


From (2.2) and (2.8), we have

$$
u(1) \geq \frac{\sum_{i=1}^{s-1} \alpha_{i}\left(\eta_{i}^{2}-\eta_{i-1}^{2}\right)+\sum_{i=s}^{m-2} \frac{\alpha_{i}}{\eta_{i}}\left(\eta_{i}^{2}-\eta_{i-1}^{2}\right)\left(1-\eta_{i}\right)}{2-\sum_{i=s}^{m-2} \alpha_{i}\left(\eta_{i}^{2}-\eta_{i-1}^{2}\right)} u(\bar{t}) .
$$

Thus, $\inf _{t \in\left[\eta_{1}, 1\right]} u(t) \geq \Gamma\|u\|$.

Step 4. If $\bar{t}>\eta_{m-2}$ and $\inf _{t \in\left[\eta_{1}, 1\right]} u(t)=u(1)$, for $1 \leq i \leq m-2$,

$$
u\left(\eta_{i}\right) \geq \eta_{i} u(\bar{t})
$$

Then, we obtain

$$
\frac{1}{2} \sum_{i=1}^{m-2} \frac{\alpha_{i}}{\eta_{i}}\left(\eta_{i}^{2}-\eta_{i-1}^{2}\right) u\left(\eta_{i}\right) \geq \frac{1}{2} \sum_{i=1}^{m-2} \alpha_{i}\left(\eta_{i}^{2}-\eta_{i-1}^{2}\right) u(\bar{t})
$$

From (2.8),

$$
u(1) \geq \frac{1}{2} \sum_{i=1}^{m-2} \alpha_{i}\left(\eta_{i}^{2}-\eta_{i-1}^{2}\right) u(\bar{t})
$$

Thus, $\inf _{t \in\left[\eta_{1}, 1\right]} u(t) \geq \Gamma\|u\|$.

2.4. Lemma. Assume that $(C 1)$ and $(C 2)$ hold, then for all $t \in[0,1]$, the following result is true.

$$
u(t) \geq \frac{\delta}{d} \int_{\eta_{m-2}}^{1}(1-s) y(s) d s, \quad t \in[0,1]
$$

where

$$
\delta=\min \left\{2 \gamma\left(1-\sum_{i=1}^{m-1} \alpha_{i}\right), \gamma+\beta \sum_{i=1}^{m-1} \alpha_{i}\left(\eta_{i}^{2}-\eta_{i-1}^{2}\right)\right\} .
$$

Proof. From the fact that $u^{\prime \prime}(t)=-y(t) \leq 0$, we know that the graph of $u$ is concave down on $[0,1]$. Then, By Lemma 2.2, one can easily prove that,

$$
u(t) \geq \frac{\delta}{d} \int_{\eta_{m-2}}^{1}(1-s) y(s) d s \quad t \in[0,1]
$$

Let $\mathbb{B}=\mathcal{C}[0,1]$ is a Banach space with the norm $\|u\|=\max _{t \in[0,1]}|u(t)|$. Define the cone $K \subset \mathbb{B}$ by

$$
K=\left\{u \in \mathbb{B}: u(t) \geq 0 \text { for } t \in[0,1] \text { and } \min _{t \in\left[\eta_{1}, 1\right]} u(t) \geq \Gamma\|u\|\right\},
$$

where the number $\Gamma$ be given as in equation (2.4). We can define an operator $T: K \rightarrow \mathbb{B}$ by 


$$
\begin{aligned}
T u(t)= & -\int_{0}^{t}(t-s) f(s, u(s)) d s+\frac{2(\gamma+\beta t)}{d} \int_{0}^{1}(1-s) f(s, u(s)) d s \\
& -\frac{\gamma+\beta t}{d} \sum_{i=1}^{m-1} \alpha_{i} \int_{0}^{\eta_{i}}\left(\eta_{i}-s\right)^{2} f(s, u(s)) d s \\
& +\frac{\gamma+\beta t}{d} \sum_{i=1}^{m-1} \alpha_{i} \int_{0}^{\eta_{i-1}}\left(\eta_{i-1}-s\right)^{2} f(s, u(s)) d s,
\end{aligned}
$$

for $u \in K$. Obviously, $u$ is the solution of the BVP (1.1) if and only if $u$ is a fixed point of $T$.

2.5. Lemma. Let $(C 1)$ and $(C 2)$ hold. Then $T: K \rightarrow K$ is completely continuous and $T(K) \subset K$.

Proof. Define the operator $T: K \rightarrow K$ by

$$
\begin{aligned}
T u(t)= & -\int_{0}^{t}(t-s) f(s, u(s)) d s+\frac{2(\gamma+\beta t)}{d} \int_{0}^{1}(1-s) f(s, u(s)) d s \\
& -\frac{\gamma+\beta t}{d} \sum_{i=1}^{m-1} \alpha_{i} \int_{0}^{\eta_{i}}\left(\eta_{i}-s\right)^{2} f(s, u(s)) d s \\
& +\frac{\gamma+\beta t}{d} \sum_{i=1}^{m-1} \alpha_{i} \int_{0}^{\eta_{i-1}}\left(\eta_{i-1}-s\right)^{2} f(s, u(s)) d s
\end{aligned}
$$

for $u \in K$. By Lemma 2.2, for all $u \in K$ we have $T u \geq 0$, and Lemma 2.3, $T(K) \subset K$. Next, by standard methods and Arzela-Ascoli theorem, one can easily prove that operator $\mathrm{T}$ is completely continuous.

Now for convenience, we introduce the following notations. Let

$$
\begin{gathered}
L=\left[\left(2+\sum_{i=1}^{m-1} \alpha_{i}\right) \frac{\gamma+\beta}{d} \int_{0}^{1}(1-s) d s\right]^{-1} \\
M=\left[\frac{1}{d} \int_{\eta_{m-2}}^{1}(1-s) d s\right]^{-1}, \quad \psi=\frac{\delta}{M} .
\end{gathered}
$$

We assume $0<\psi \leq \Gamma<1$. The numbers $\Gamma$ and $\delta$ are given by (2.4), (2.9).

In order to follow the main results of this paper easily, now we state the fixed point theorems which we applied to prove Theorems 3.1 and 3.3.

2.6. Theorem. ([8]) Let $K$ be a cone in a real Banach space $\mathbb{B}$. Let $D$ be an open bounded subset of $\mathbb{B}$ with $D_{K}=D \cap K \neq \emptyset$ and $\bar{D}_{K} \neq K$. Assume that $T: \bar{D}_{K} \rightarrow K$ is completely continuous such that $u \neq T u$ for $u \in \partial D_{K}$. Then the following results hold:

(i) If $\|T u\| \leq\|u\|, u \in \partial D_{K}$, then $i_{K}\left(T, D_{K}\right)=1$;

(ii) If there exists $e \in K \backslash\{0\}$ such that $u \neq T u+\lambda e$ for all $u \in \partial D_{K}$ and all $\lambda>0$, then $i_{K}\left(T, D_{K}\right)=0$;

(iii) Let $U$ be open in $K$ such that $\bar{U} \subset D_{K}$. If $i_{K}\left(T, D_{K}\right)=1$ and $i_{K}\left(T, U_{K}\right)=0$, then $T$ has a fixed point in $D_{K} \backslash \bar{U}_{K}$. The same result holds if $i_{K}\left(T, D_{K}\right)=0$ and $i_{K}\left(T, U_{K}\right)=1$. 
We define

$$
\begin{aligned}
& K_{\rho}=\{u \in K:\|u\|<\rho\}, \\
& \Omega_{\rho}=\left\{u \in K: \min _{t \in\left[\eta_{1}, 1\right]} u(t)<\Gamma \rho\right\}=\left\{u \in K: \Gamma\|u\| \leq \min _{t \in\left[\eta_{1}, 1\right]} u(t)<\Gamma \rho\right\} .
\end{aligned}
$$

2.7. Lemma. $\Omega_{\rho}$ has the following properties:

(a) $\Omega_{\rho}$ is open relative to $K$.

(b) $K_{\Gamma \rho} \subset \Omega_{\rho} \subset K_{\rho}$.

(c) $u \in \partial \Omega_{\rho}$ if and only if $\min _{t \in\left[\eta_{1}, 1\right]} u(t)=\Gamma \rho$.

(d) If $u \in \partial \Omega_{\rho}$, then $\Gamma \rho \leq u(t) \leq \rho$ for $t \in\left[\eta_{1}, 1\right]$.

2.8. Theorem. ([8]) Let $\mathbb{B}$ be a Banach space, $K \subseteq \mathbb{B}$ a cone of $\mathbb{B}$. Set

$$
\begin{aligned}
K_{r} & =\{u \in K:\|u\|<r\}, \\
P(\varphi, a, b) & =\{u \in K: a \leq \varphi(u),\|u\| \leq b\} .
\end{aligned}
$$

Suppose $T: \overline{K_{r}} \rightarrow \overline{K_{r}}$ be a completely continuous operator and $\varphi$ be a nonnegative, continuous, concave functional on $K$ with $\varphi(u) \leq\|u\|$ for all $u \in \overline{K_{r}}$. If there exists $0<p<q<d \leq r$ such that the following conditions hold:

(i) $\{u \in K(\varphi, q, d): \varphi(u)>q\} \neq \emptyset$ and $\varphi(T u)>q$ for all $u \in K(\varphi, q, d)$;

(ii) $\|T u\|<p$ for all $\|u\| \leq p$;

(iii) $\varphi(T u)>q$ for $u \in K(\varphi, q, r)$ with $\|T u\|>d$.

Then $\mathrm{T}$ has at least three positive solutions $u_{1}, u_{2}$ and $u_{3}$ in $\overline{K_{r}}$ satisfying

$$
\left\|u_{1}\right\|<p, \varphi\left(u_{2}\right)>q, \quad p<\left\|u_{3}\right\| \text { with } \varphi\left(u_{3}\right)<q .
$$

\section{Main results}

In this section, we will prove the existence of at least two and three positive solutions of the BVP (1.1). The following theorems we will make use of the fixed-point index theorem and the Leggett-Williams fixed point theorem, respectively.

We define,

$$
\begin{aligned}
f_{\Gamma \rho}^{\rho} & =\min \left\{\min _{t \in\left[\eta_{1}, 1\right]} \frac{f(t, u)}{\rho}: u \in[\Gamma \rho, \rho]\right\}, \\
f_{0}^{\rho} & =\max \left\{\max _{t \in[0,1]} \frac{f(t, u)}{\rho}: u \in[0, \rho]\right\} .
\end{aligned}
$$

3.1. Theorem. Suppose $(C 1)$ and $(C 2)$ hold.

(C3) There exist $\rho_{1}, \rho_{2}, \rho_{3} \in(0, \infty)$ with $\rho_{1}<\Gamma \rho_{2}$ and $\rho_{2}<\rho_{3}$ such that

$$
f_{0}^{\rho_{1}} \leq L, f_{\Gamma \rho_{2}}^{\rho_{2}} \geq \frac{\Gamma M}{\delta}, u \neq T u \text { for } u \in \partial \Omega_{\rho_{2}} \text { and } f_{0}^{\rho_{3}} \leq L .
$$

Then the BVP (1.1) has at least two positive solutions $u_{1}$, $u_{2}$ with $u_{1} \in \Omega_{\rho_{2}} \backslash \bar{K}_{\rho_{1}}, u_{2} \in$ $K_{\rho_{3}} \backslash \bar{\Omega}_{\rho_{2}}$.

(C4) There exist $\rho_{1}, \rho_{2}, \rho_{3} \in(0, \infty)$ with $\rho_{1}<\rho_{2}<\Gamma \rho_{3}<\rho_{3}$ such that

$$
f_{\Gamma \rho_{1}}^{\rho_{1}} \geq \frac{\Gamma M}{\delta}, f_{0}^{\rho_{2}} \leq L, u \neq \text { Tu for } u \in \partial K_{\rho_{2}} \text { and } f_{\Gamma \rho_{3}}^{\rho_{3}} \geq \frac{\Gamma M}{\delta} .
$$

Then the $B V P(1.1)$ has at least two positive solutions $u_{1}, u_{2}$ with $u_{1} \in K_{\rho_{2}} \backslash \bar{\Omega}_{\rho_{1}}, u_{2} \in$ $\Omega_{\rho_{3}} \backslash \bar{K}_{\rho_{2}}$. 
Proof. We only consider the condition $(C 3)$. If $(C 4)$ holds, then the proof is similar to that of the case when $(C 3)$ holds. By Lemma 2.5, we know that the operator $T: K \rightarrow K$ is completely continuous.

Firstly, we show that $i_{K}\left(T, K_{\rho_{1}}\right)=1$. In fact, by $(2.10)$ and $f_{0}^{\rho_{1}} \leq L$, we have for $u \in \partial K_{\rho_{1}}$,

$$
\begin{gathered}
\|T u(t)\|=\max _{t \in[0,1]} \mid-\int_{0}^{t}(t-s) f(s, u(s)) d s+\frac{2(\gamma+\beta t)}{d} \int_{0}^{1}(1-s) f(s, u(s)) d s \\
\quad-\frac{\gamma+\beta t}{d} \sum_{i=1}^{m-1} \alpha_{i} \int_{0}^{\eta_{i}}\left(\eta_{i}-s\right)^{2} f(s, u(s)) d s \\
\quad+\frac{\gamma+\beta t}{d} \sum_{i=1}^{m-1} \alpha_{i} \int_{0}^{\eta_{i-1}}\left(\eta_{i-1}-s\right)^{2} f(s, u(s)) d s \mid \\
\leq \quad \frac{2(\gamma+\beta t)}{d} \int_{0}^{1}(1-s) f(s, u(s)) d s \\
\quad+\frac{\gamma+\beta t}{d} \sum_{i=1}^{m-1} \alpha_{i} \int_{0}^{1}(1-s) f(s, u(s)) d s \\
\leq \quad\left(2+\sum_{i=1}^{m-1} \alpha_{i}\right) \frac{\gamma+\beta}{d} \int_{0}^{1}(1-s) f(s, u(s)) d s \\
\leq \rho_{1}
\end{gathered}
$$

i.e., $\|T u\| \leq\|u\|$ for $u \in \partial K_{\rho_{1}}$. By $(i)$ of Theorem 2.6, we obtain that $i_{K}\left(T, K_{\rho_{1}}\right)=1$.

Secondly, we show that $i_{K}\left(T, \Omega_{\rho_{2}}\right)=0$. Let $e(t) \equiv 1$. Then $e \in \partial K_{1}$. We claim that

$$
u \neq T u+\lambda e, u \in \partial \Omega_{\rho_{2}}, \lambda>0 .
$$

Suppose that there exists $u_{0} \in \partial \Omega_{\rho_{2}}$ and $\lambda_{0}>0$ such that

$$
u_{0}=T u_{0}+\lambda_{0} e .
$$

Then, Lemma 2.4, (2.10) and (3.1) imply that for $t \in\left[\eta_{1}, 1\right]$

$$
\begin{aligned}
u_{0}= & T u_{0}+\lambda_{0} e \\
= & -\int_{0}^{t}(t-s) f(s, u(s)) d s+\frac{2(\gamma+\beta t)}{d} \int_{0}^{1}(1-s) f(s, u(s)) d s \\
& -\frac{\gamma+\beta t}{d} \sum_{i=1}^{m-1} \alpha_{i} \int_{0}^{\eta_{i}}\left(\eta_{i}-s\right)^{2} f(s, u(s)) d s \\
& +\frac{\gamma+\beta t}{d} \sum_{i=1}^{m-1} \alpha_{i} \int_{0}^{\eta_{i-1}}\left(\eta_{i-1}-s\right)^{2} f(s, u(s)) d s+\lambda_{0} e \\
\geq & \frac{\delta}{d} \int_{\eta_{m-2}}^{1}(1-s) f(s, u(s)) d s+\lambda_{0} \\
= & \Gamma \rho_{2}+\lambda_{0}
\end{aligned}
$$

i.e., $\Gamma \rho_{2} \geq \Gamma \rho_{2}+\lambda_{0}$, which is a contradiction. Hence by $(i i)$ of Theorem 2.6, it follows that $i_{K}\left(T, \Omega_{\rho_{2}}\right)=0$.

Finally, similar to the proof of $i_{K}\left(T, K_{\rho_{1}}\right)=1$, we can prove that $i_{K}\left(T, K_{\rho_{3}}\right)=1$. Since $\rho_{1}<\Gamma \rho_{2}$ and Lemma $2.7(b)$, we have $\bar{K}_{\rho_{1}} \subset K_{\Gamma \rho_{2}} \subset \Omega_{\rho_{2}}$. Similarly with $\rho_{2}<\rho_{3}$ and Lemma $2.7(b)$, we have $\bar{\Omega}_{\rho_{2}} \subset K_{\rho_{2}} \subset K_{\rho_{3}}$. Therefore (iii) of Theorem 2.6 implies that BVP (1.1) has at least two positive solutions $u_{1}, u_{2}$ with $u_{1} \in \Omega_{\rho_{2}} \backslash \bar{K}_{\rho_{1}}, u_{2} \in$ $K_{\rho_{3}} \backslash \bar{\Omega}_{\rho_{2}}$. 
3.2. Example. We consider the following $m$-point integral boundary value problem

$$
\left\{\begin{array}{l}
u^{\prime \prime}(t)+f(t, u(t))=0, \quad t \in(0,1), \\
u^{\prime}(0)=0, \quad u(1)=\frac{1}{3} \int_{0}^{\frac{1}{6}} u(s) d s+\frac{1}{4} \int_{\frac{2}{6}}^{\frac{3}{6}} u(s) d s+\frac{1}{6} \int_{\frac{4}{6}}^{\frac{5}{6}} u(s) d s,
\end{array}\right.
$$

where

$$
f(t, u)= \begin{cases}\frac{\sin t}{200}+0.02, & u \leq 0.0204 \\ \frac{\sin t}{200}+\frac{4400}{7} u-\frac{8962}{700}, & 0.0204 \leq u \leq 0.0904 \\ \frac{\sin t}{200}+\frac{5}{6} u+\frac{65917}{1500}, & u \geq 0.0904\end{cases}
$$

Set $\beta=0, \gamma=1, m=7, \eta_{i}=\frac{i}{6} \quad$ for $i=0, \ldots, 6, \alpha_{j}=0$ for $j=2,4,6$, and $\alpha_{1}=\frac{1}{3}, \alpha_{3}=\frac{1}{4}, \alpha_{5}=\frac{1}{6}$. By simple calculation, we get $d=\frac{7}{4}, L=\frac{14}{11}, M=126$, $\delta=\frac{37}{142}, \Gamma=\frac{113}{4135}$. It is clear that conditions $(C 1)$ and $(C 2)$ are satisfied. Taking $\rho_{1}=0.0204, \rho_{2}=3.308, \rho_{3}=169.02$, we can obtain that

$$
\rho_{1}<\Gamma \rho_{2} \quad \text { and } \quad \rho_{2}<\rho_{3} .
$$

Now, we show that $(C 3)$ is satisfied:

$$
\begin{aligned}
f_{0}^{0.0204} & \leq 0.025<L, \\
f_{0.0904}^{3.308} & \geq 44.02>\frac{\Gamma M}{\delta}, \\
f_{0}^{169.02} & \leq 184.794<L .
\end{aligned}
$$

Then, (C3) condition of Theorem 3.1 holds. Hence, we get the BVP (3.2) has at least two positive solutions.

We now give the sufficient conditions to have at least three non-negative solutions for the BVP (1.1). Firstly, we define the nonnegative, continuous, concave functional $\varphi: K \rightarrow[0,+\infty)$ by

$$
\varphi(u)=\min _{t \in\left[\eta_{1}, 1\right]}|u(t)|, \quad \forall u \in K .
$$

It is obvious that $\varphi(u) \leq\|u\|$ for all $u \in K$.

3.3. Theorem. Suppose $(C 1)$ and $(C 2)$ hold. Moreover there exist nonnegative numbers $0<p<q \leq \min \{\psi, \psi L\} r$ such that

(i) $f(t, u) \leq L r$ for $t \in[0,1]$ and $u \in[0, r]$;

(ii) $f(t, u)>\frac{q}{\psi}$ for $t \in\left[\eta_{1}, 1\right]$ and $u \in\left[q, \frac{q}{\psi}\right]$;

(iii) $f(t, u)<L p$ for $t \in[0,1]$ and $u \in[0, p]$.

Then the BVP (1.1) has at least three non-negative solutions $u_{1}, u_{2}$ and $u_{3}$ satisfying

$$
\left\|u_{1}\right\|<p, \varphi\left(u_{2}\right)>q, \quad p<\left\|u_{3}\right\| \text { with } \varphi\left(u_{3}\right)<q .
$$


Proof. The conditions of Theorem 2.8 will be shown to be satisfied. If $u \in \overline{K_{r}}$ then $\|u\| \leq r$ and assumption (i) we have

$$
\begin{aligned}
\|T u(t)\|=\max _{t \in[0,1]} \mid-\int_{0}^{t}(t-s) f(s, u(s)) d s+\frac{2(\gamma+\beta t)}{d} \int_{0}^{1}(1-s) f(s, u(s)) d s \\
\quad-\frac{\gamma+\beta t}{d} \sum_{i=1}^{m-1} \alpha_{i} \int_{0}^{\eta_{i}}\left(\eta_{i}-s\right)^{2} f(s, u(s)) d s \\
\quad+\frac{\gamma+\beta t}{d} \sum_{i=1}^{m-1} \alpha_{i} \int_{0}^{\eta_{i-1}}\left(\eta_{i-1}-s\right)^{2} f(s, u(s)) d s \mid \\
\leq \quad \frac{2(\gamma+\beta t)}{d} \int_{0}^{1}(1-s) f(s, u(s)) d s \\
\quad+\frac{\gamma+\beta t}{d} \sum_{i=1}^{m-1} \alpha_{i} \int_{0}^{1}(1-s) f(s, u(s)) d s \\
\leq \quad\left(2+\sum_{i=1}^{m-1} \alpha_{i}\right) \frac{\gamma+\beta}{d} \int_{0}^{1}(1-s) f(s, u(s)) d s \\
\leq \quad r .
\end{aligned}
$$

Therefore, $T: \overline{K_{r}} \rightarrow \overline{K_{r}}$. By (iii) and the argument above, we can get that $T: \overline{K_{p}} \rightarrow K_{p}$.

Now, we show that the condition $(i)$ of Theorem 2.8 is satisfied. Since $\frac{q}{\psi} \in K\left(\varphi, q, \frac{q}{\psi}\right)$ and $\varphi\left(\frac{q}{\psi}\right)=\frac{q}{\psi}>q,\left\{u \in K\left(\varphi, q, \frac{q}{\psi}\right): \varphi(u)>q\right\} \neq \emptyset$. If $u \in K\left(\varphi, q, \frac{q}{\psi}\right)$, then $q \leq u(t) \leq \frac{q}{\psi}$ for $t \in\left[\eta_{1}, 1\right]$. From assumption (ii), we have $f(t, u)>\frac{q}{\psi}$ for $t \in\left[\eta_{1}, 1\right]$, and by the conditions of $\varphi$ and the operator $T$, we have to distinguish two cases,

(i) $\varphi(T u)=(T u)\left(\eta_{1}\right)$, and

(ii) $\varphi(T u)=(T u)(1)$.

In Case (i), we have

$$
\begin{aligned}
\varphi(T u)= & (T u)\left(\eta_{1}\right) \\
= & \frac{1}{d} \int_{0}^{\eta_{1}}\left[2(\gamma+\beta s)\left[\left(1-\eta_{1}\right)-\sum_{i=2}^{m-1} \alpha_{i}\left(\eta_{i}^{2}-\eta_{i-1}^{2}\right)+\sum_{i=2}^{m-1} \eta_{1} \alpha_{i}\left(\eta_{i}-\eta_{i-1}\right)\right]\right. \\
& \left.+\eta_{1} \beta \alpha_{1} s\left(\eta_{1}-s\right)+\gamma \alpha_{1}\left(\eta_{1}^{2}-s^{2}\right)\right] f(s, u(s)) d s \\
& +\frac{\left(\gamma+\beta \eta_{1}\right)}{d} \sum_{i=2}^{m-2} \alpha_{i} \int_{\eta_{i-1}}^{\eta_{i}}\left[2(1-s)-\sum_{j=i}^{m-2} \alpha_{j}\left(\eta_{j}-s\right)^{2}\right. \\
& \left.+\sum_{j=i+1}^{m-2} \alpha_{j}\left(\eta_{j-1}-s\right)\right] f(s, u(s)) d s \\
& +\frac{2\left(\gamma+\beta \eta_{1}\right)}{d} \int_{\eta_{m-2}}^{1}(1-s) f(s, u(s)) d s
\end{aligned}
$$




$$
\begin{aligned}
& \geq \frac{2\left(\gamma+\beta \eta_{1}\right)}{d} \int_{\eta_{m-2}}^{1}(1-s) f(s, u(s)) d s \\
& \geq \frac{2 \gamma\left(1-\sum_{i=1}^{m-1} \alpha_{i}\right)}{d} \int_{\eta_{m-2}}^{1}(1-s) f(s, u(s)) d s \\
& \geq \frac{\delta}{d} \int_{\eta_{m-2}}^{1}(1-s) f(s, u(s)) d s \\
& >q .
\end{aligned}
$$

In Case (ii), we get

$$
\begin{aligned}
\varphi(T u) & =(T u)(1) \\
& \geq \frac{\gamma+\beta}{d} \sum_{i=1}^{m-1} \alpha_{i}\left(\eta_{i}^{2}-\eta_{i-1}^{2}\right) \int_{\eta_{m-2}}^{1}(1-s) f(s, u(s)) d s \\
& \geq \frac{\delta}{d} \int_{\eta_{m-2}}^{1}(1-s) f(s, u(s)) d s \\
& >q .
\end{aligned}
$$

i.e.,

$$
\varphi(T u)>q, \text { for all } u \in K\left(\varphi, q, \frac{q}{\psi}\right) .
$$

Hence, condition (i) of Theorem 2.8 is satisfied.

Finally, we show that condition (iii) of Theorem 2.8 is also satisfied. If $u \in K(\varphi, q, r)$ and $\|T u\|>\frac{q}{\psi}$ then

$$
\varphi(T u)=\min _{t \in\left[\eta_{1}, 1\right]}|T u(t)| \geq \Gamma|| T u \|>\Gamma \frac{q}{\psi} \geq q .
$$

Therefore, condition (iii) of Theorem 2.8 is also satisfied. Since all conditions of Theorem 2.8 are verified, the BVP (1.1) has at least three non-negative solutions such that

$$
\left\|u_{1}\right\|<p, \quad q \leq \varphi\left(u_{2}\right) \text { and } p<\left\|u_{3}\right\| \text { with } \varphi\left(u_{3}\right)<q .
$$

3.4. Example. We consider the following second-order boundary value problem,

$$
\left\{\begin{array}{l}
u^{\prime \prime}(t)+\frac{2005 u^{4}}{u^{4}+2006}=0, \quad t \in(0,1), \\
u(0)-u^{\prime}(0)=0, \quad u(1)=\frac{1}{2} \int_{0}^{\frac{1}{4}} u(s) d s+\frac{1}{3} \int_{\frac{1}{2}}^{1} u(s) d s .
\end{array}\right.
$$

When we take $\beta=\gamma=1, m=4, \eta_{0}=0, \eta_{1}=\frac{1}{4}, \eta_{2}=\frac{1}{2}, \eta_{3}=1$ and $\alpha_{1}=\frac{1}{2}, \alpha_{2}=0$, $\alpha_{3}=\frac{1}{3}$, we obtain $d=\frac{301}{96}, L=\frac{301}{272}, M=\frac{301}{12}, \Gamma=\frac{1}{64}, \psi=\frac{4}{301}$.

$$
f(t, u)=f(u)=\frac{2005 u^{4}}{u^{4}+2006}
$$

is continuous and increasing on $[0,+\infty)$. Now, we check that the conditions of Theorem 3.3 are satisfied. When we choose $r=2720$, from $\lim _{u \rightarrow \infty} f(u)=2005$, we obtain 


$$
f(u) \leq 2005<L r=3010, \quad u \in[0, r] .
$$

Consequently, the condition (i) of Theorem 3.3 is satisfied. As $f(10) \approx 1669.99$, we get

$$
f(u)>\frac{q}{\psi}=752.5, \quad u \in\left[q, \frac{q}{\psi}\right] .
$$

It means that condition (ii) of Theorem 3.3 is satisfied. Finally, let $p=1$, as $f(1) \approx 0.999$, we get

$$
f(u)<L p \approx 1.106, \quad u \in[0, p],
$$

so that condition (iii) of Theorem 3.3 is satisfied. Therefore, there exist numbers $p=$ $1, q=10, r=2720$ satisfying

$$
0<p<q \leq \min \{\psi, \psi L\} r \text { and } 0<\psi<\Gamma<1
$$

such that all the conditions of Theorem 3.3 hold. So the boundary value problem has at least three positive solutions $u_{1}, u_{2}$ and $u_{3}$ satisfying

$$
\left\|u_{1}\right\|<1, \quad 10 \leq \min _{t \in\left[\eta_{1}, 1\right]} u_{2}(t) \text { and } 1<\left\|u_{3}\right\| \text { with } \min _{t \in\left[\eta_{1}, 1\right]} u_{3}(t)<10 .
$$

\section{References}

[1] Agarwal, R. P., O'Regan, D., Infinite Interval Problems for Differential, Difference and Integral Equations, Kluwer Academic Publishers, Dordrecht, The Netherlands, 2001.

[2] Bitsadze, A. V., Samarskii, A. A., Some elementary generalizations of linear elliptic boundary value problems (in Russian), Anal. Dokl. Akad. Nauk SSSR, 185(1969), 739-740, English translation: Soviet Math. Dokl. 10, 398-400, 1969.

[3] Boucherif, A., Second-order boundary value problems with integral boundary conditions, Nonlinear Anal. 70 , 364-371, 2009.

[4] Conti, R., Recent trends in the theory of boundary value problems for ordinary differential equations, Boll. Un. Mat. Ital. 22, 135-178, 1967.

[5] Corduneanu, C., Integral Equations and Applications, Cambridge University Press, Cambridge, UK, 1991.

[6] Feng, M., Ji, D., Ge, W., Positive solutions for a class of boundary-value problem with integral boundary conditions in Banach spaces, J. Comput. Appl. Math. 222, 351-363, 2008.

[7] Gallardo, J. M., Second-order differential operators with integral boundary conditions and generation of analytic semigroups, Rocky Mountain J. Math. 30, 1265-1291, 2000.

[8] Guo, D., Lashmikanthan, V., Nonlinear Problems in Abstract Cones, Academic Press, San Diego, 1988.

[9] Il'in, V. A., Moiseev, E. I., Nonlocal boundary value problem of the second kind for a SturmLiouville operator, Differ. Equ. 23, 979-987, 1987.

[10] Jankowski, T., Positive solutions for fourth-order differential equations with deviating arguments and integral boundary conditions, Nonlinear Anal. 73, 1289-1299, 2010.

[11] Ntouyas, S. K., Nonlocal initial and boundary value problems: a survey, in: Handbook of differential equations: ordinary differential equations, Vol. II, Elsevier B. V., Amsterdam, 461-557, 2005.

[12] Picone, M., Su un problema al contorno nelle equazioni differenziali lineari ordinarie del secondo ordine (in Italian), Ann. Scuola Norm. Sup. Pisa Cl. Sci. 10, 1-95, 1908.

[13] Tariboon, J., Sudsutad, W., Positive solutions of a nonlinear m-point integral boundary value problem, Appl. Math. Sci. 6, 711-729, 2012.

[14] Webb, J. R. L., Infante, G., Positive solutions of nonlocal boundary value problems involving integral conditions, NoDEA Nonlinear Differential Equations Appl. 15, 45-67, 2008. 
[15] Webb, J. R. L., Infante, G., Positive solutions of nonlocal boundary value problems: a unified approach, J. London Math. Soc. 74, 673-693, 2006.

[16] Whyburn, W. M. Differential equations with general boundary conditions, Bull. Amer. Math. Soc. 48, 692-704, 1942.

[17] Yoruk, F., Hamal, N. A., Existence results for nonlinear boundary value problems with integral boundary conditions on an infinite interval, Bound. Value Probl., 2012:127, 17 pp., 2012. doi:10.1186/1687-2770-2012-127.

[18] Zhang, X, Feng, M., Ge, W., Existence results for nonlinear boundary-value problems with integral boundary conditions in Banach spaces, Nonlinear Anal. 69, 3310-3321, 2008. 